DOI: https://doi.org/10.46296/rc.v5i9.0037

\title{
Gestión de Comunicación Externa para el posicionamiento de la actividad económica en la Parroquia Atahualpa
}

\section{External Communication Management for the positioning of the economic activity in the Atahualpa Parish}

\author{
Yagual-Orrala Yulady Inés \\ Universidad Estatal Península de Santa Elena. Santa Elena, Ecuador. \\ Correo: yulady.yagualorrala@upse.edu.ec \\ ORCID ID: https://orcid.org/0000-0001-6423-9537 \\ Iza-Espinoza Luis Alberto \\ Universidad Estatal Península de Santa Elena. Santa Elena, Ecuador. \\ Correo: liza@upse.edu.ec \\ ORCID ID: https://orcid.org/0000-0002-0522-9723
}

\begin{abstract}
RESUMEN
La presente investigación sobre: Gestión de Comunicación Externa para el posicionamiento de la actividad económica en la Parroquia Atahualpa, tiene como objetivo: Analizar la Gestión de Comunicación Externa para el posicionamiento de la actividad económica en la Parroquia Atahualpa. Para este proceso se aplicó la siguiente metodología: Investigación básica, diseño de investigación descriptiva, fuente de investigación documental, método inductivo, la población y muestra está determinada por el cuerpo administrativo del GAD Parroquial conformado por nueve miembros en diferentes áreas. Se aplicó la técnica de encuesta a través del cuestionario como instrumento para recolección de información en las que se obtuvo los siguientes resultados: Se identificaron gestiones comunicacionales externas empleadas por el GAD Parroquial como la utilización de estrategias para lograr posicionarse económicamente a través de sus actividades. Con respecto a la discusión se corrobora que las gestiones son manejadas por el cuerpo administrativo del GAD Parroquial y no por profesionales en comunicación. Finalmente, la conclusión corresponde al uso de redes sociales como medio para difundir información, la elaboración y creación de publicidades de las actividades comerciales de los habitantes de la parroquia, también los vínculos que tiene con otras instituciones para el mejoramiento de proyectos y planificaciones laborales.
\end{abstract}

Palabras claves: Gestiones externas, posicionamiento, economía, comunicación, instituciones públicas.

\begin{abstract}
The present research on: External Communication Management for the positioning of the economic activity in the Atahualpa Parish, has as objective: To analyze the External Communication Management for the positioning of the economic activity in the Atahualpa Parish. For this process the following methodology was applied: Basic research, descriptive research design, documentary research source, inductive method, the population and sample is determined by the administrative body of the GAD Parroquial formed by nine members in different Información del manuscrito:
\end{abstract}

Fecha de recepción: 01 de diciembre de 2021.

Fecha de aceptación: 27 de diciembre de 2021.

Fecha de publicación: 06 de enero de 2022. 
areas. The survey technique was applied through a questionnaire as an instrument for information gathering, in which the following results were obtained: External communication efforts used by the GAD Parroquial were identified as the use of strategies to achieve economic positioning through its activities. With respect to the discussion, it is corroborated that these efforts are managed by the administrative body of the GAD Parroquial and not by communication professionals. Finally, the conclusion corresponds to the use of social networks as a means to disseminate information, the elaboration and creation of advertisements of the commercial activities of the inhabitants of the parish, as well as the links with other institutions for the improvement of projects and work planning.

Keywords: External management, positioning, economics, communication, public institutions.

\section{INTRODUCCIÓN}

El presente trabajo investigativo estudia la Gestión de Comunicación Externa para el posicionamiento de la actividad económica en la Parroquia Atahualpa, para este proceso se describen investigaciones realizadas a nivel internacional, nacional y local las cuales sustentan la investigación. A continuación, se sugieren los siguientes autores:

Para Carlos (2020), en su estudio elaborado en la provincia de Córdoba Argentina y titulado "La gestión de la comunicación externa en A.J y J.A Redolfi SRL: El fortalecimiento del vínculo institucional con los clientes corporativos". Indica que:

Se analiza la comunicación externa desde la perspectiva profesional de las Relaciones Públicas e Institucionales con el propósito de optimizar y gestionar los vínculos con sus grupos de interés. En esta arista trata de acciones particulares como la configuración de identidad corporativa, además, se complementa este lineamiento con la gestación de espacios institucionales, donde la empresa debe estratificar sus comunicaciones para fortalecer sus relaciones y captar nuevos vínculos que logre ampliar su mercado a zonas contiguas de argentina (p.2).

En efecto la comunicación externa como soporte institucional genera lazos con el público perteneciente a una comunidad para realizar gestiones que ayuden al desarrollo social. 
El autor Gutiérrez (2015), en su investigación “La comunicación estratégica como herramienta de posicionamiento en negocios familiares. Estudio de Caso Gilisburger México". Manifiesta que:

La investigación muestra un panorama evolutivo de los medios de comunicación, dejando atrás el sentido unidireccional de la comunicación para abordar procesos de interacción y el intermediarismo. Se presenta la problemática actual en Gilisburger, que mediante un marco referencial direccionará a un enfoque de corrientes filosóficas y literarias que establecen el objeto de estudio. Por último, el proceso metodológico permitirá determinar propuestas de estrategia digital como conclusión alternativa al cambio (p.4).

Desde luego, la comunicación estratégica direccionada al incremento económico se convierte en la herramienta de gestión que son utilizadas por las empresas e instituciones.

A nivel nacional se tiene como referencia a la autora Ochoa (2019), en su artículo "Las redes sociales de la Universidad Nacional de Chimborazo como herramientas de gestión en la comunicación externa, período octubre 2017 marzo 2018". Señala que:

Las redes sociales como herramienta de gestión en la comunicación externa de la Universidad Nacional de Chimborazo, donde se realiza un diagnóstico a través del método inductivo - deductivo que describe su forma de uso, siendo su técnica la entrevista que le permite identificar hechos partiendo de aspectos generales a particulares. Los resultados obtenidos establecieron que la gestión de las redes sociales de la Universidad genera poca interacción con el público, concluyendo que las redes sociales utilizadas llegan con información directa y precisa (p.3).

Evidentemente las redes sociales como instrumento de gestión en la comunicación externa agilizan él envió de información desde la organización hacia su público objetivo. 
Por su parte el estudio de Zavala y Lasso (2017), realizada en Guayaquil sobre "Pymes como modelo económico en la creación de estrategias de comunicación". Muestra que:

Busca desarrollar un diagnóstico de las estrategias de comunicación de las Pymes en la ciudad de Guayaquil, considerada el sector que ha tenido grandes impactos por factores externos que permite el posicionamiento económico, así mismo por factores internos que tienen que ver con los costos de producción donde su diseño no está sujeta a exigencias de los demandantes. La creación de la imagen corporativa es el primer paso para emprender o gestionar actividades económicas. Por esta razón para las Pymes es un reto lograr reconocimiento dentro de un mercado tan competitivo en la que desea destacarse (p.1).

Con respecto a este tema se enfatiza al modelo económico y sus actividades basadas en las estrategias externas que aporta estabilidad e incremento comercial en una comunidad.

En el ámbito local se acentúa a la autora Cortez (2011), en su publicación "Las relaciones públicas y su incidencia en el funcionamiento de la comunicación interna y externa de AGUAPEN S.A período 2010 -2011”. Explica que:

La influencia de la comunicación es extraordinariamente aceptable en las diferentes etapas de la sociedad, quienes trabajan en instituciones públicas y están a cargo de los departamentos de comunicación en dichas entidades, deben buscar mecanismos que les permitan relacionarse y conocer las necesidades de la gente. Es un trabajo teórico documentado y sistemático sobre las relaciones públicas y el funcionamiento de la comunicación interna y externa. Aguapen S.A debe considerar la permanente comunicación a través de códigos comunicativos establecidos con la finalidad de obtener del público la aceptación que aprobará un cambio formidable en la imagen organizacional (p.1).

Así mismo, se incluye a las relaciones públicas dentro de las gestiones comunicacionales que emplea una institución para crear interacción y confianza con otras organizaciones. 
Por otro lado, se describe la investigación de Suárez (2012), sobre "Las relaciones públicas y su influencia en la sociabilidad comunicativa entre el Gobierno Autónomo Descentralizado Parroquial de Chanduy y sus Comunidades durante el período 2011 - 2012". Especifica que:

El propósito de la investigación es conocer cómo se maneja la comunicación organizacional y la transmisión de noticias generadas desde la Parroquia Chanduy y sus comunidades, que mediante la encuesta se determinará el problema que viven las 16 comunidades y así mismo establecer las soluciones, donde los únicos beneficiarios serán los moradores; destacando la importancia de la comunicación entre los sectores y principalmente con el Gobierno Autónomo Descentralizado donde se permitirá mejorar el estilo de vida (p.2).

Se evidencia que la comunicación dentro de las comunidades en desarrollo es trascendental porque ayuda al crecimiento de los diferentes sectores específicamente al ámbito económico.

El proceso investigativo de Gestión de Comunicación Externa para el posicionamiento de la actividad económica en la Parroquia Atahualpa, donde es necesario especificar las gestiones empleadas por el GAD Parroquial, institución representativa de la comunidad, que es la encargada de realizar gestiones externas comunicacionales importantes para obtener posicionamiento económico a través de las actividades productivas, con el propósito de lograr estabilidad económica, siendo los habitantes los únicos beneficiarios en el que se logra mayor alcance en sus proyecciones direccionadas al sector primario (ganadería, artesanía, agricultura). Además, son pertinentes para generar productividad y compromiso social, se conoce que la Parroquia Atahualpa tiene un nivel económico medio y es factible determinar las causas a nivel comunicacional considerando al GAD Parroquial Atahualpa como el objeto de estudio.

De la misma forma, el investigador plantea la siguiente problemática: ¿Cuáles son las gestiones de comunicación externa para el posicionamiento de la actividad económica en la Parroquia Atahualpa?, que mediante esta interrogante 
se establecerán los objetivos para desarrollar y guiar la investigación elaborada en el Gobierno Autónomo Descentralizado (GAD) de Atahualpa.

La investigación traza como Objetivo General analizar la Gestión de Comunicación Externa para el posicionamiento de la actividad económica en la Parroquia Atahualpa; los Objetivos Específicos se detallan a continuación: Identificar las Gestiones Externas empleadas en la Parroquia Atahualpa para luego determinar el posicionamiento de la actividad económica. Además, la hipótesis sugerida en la investigación señala que la Gestión de Comunicación Externa utilizada por el GAD Parroquial Atahualpa permite alcanzar posicionamiento económico a través de sus actividades y planificaciones laborales donde estas son consumadas en conjunto con sus habitantes.

Así mismo, dentro del estudio se analizan las conceptualizaciones de las variables dependiente e independiente que son: Gestión de Comunicación Externa y el Posicionamiento de la Actividad Económica, donde el soporte de esta investigación se detalla en un marco teórico que describe las variables con sus respectivas dimensiones.

En relación con la primera variable de Gestión de Comunicación Externa se resaltan a los siguientes teóricos:

Caceres y Raymundo (2019), sugieren que "La comunicación externa la empresa o institución ha de conocer el entorno general tanto político, social, cultural y económico, pero también debe conocer las tendencias de comportamiento que prevalecen en la sociedad donde quiere hacerse conocer" (p.36). Sin embargo, en el aspecto económico es donde mayor trascendencia posee porque las gestiones externas que emplea una institución dentro de la comunidad favorecen directamente a los habitantes.

Dentro de la dimensión de la comunicación externa se halla la comunicación estratégica que "Es la práctica que tiene como objetivo convertir el vínculo de las organizaciones con su entorno cultural, social y político en una relación armoniosa y positiva desde el punto de vista de sus intereses u objetivos" (Tironi y Cavallo, 2007, p.33). La comunicación estratégica apunta a una audiencia más extensa de los cuales la organización no mantiene una interacción con los líderes de opinión, la prensa, las autoridades y las comunidades. 
American Marketing Asociation define a la publicidad como la "Colocación de avisos y mensajes persuasivos, en tiempo o espacio, comprado en cualquiera de los medios de comunicación por empresas lucrativas, organizaciones no lucrativas, agencias de estado y los individuos que intentan informar y persuadir a los miembros de un mercado" (Thompson, 2005, p.1). Es decir, la publicidad es una herramienta poderosa para que las instituciones puedan promocionar todo tipo de actividades comerciales logrando tener mayor alcance comunicacional.

Por consiguiente, la dimensión de las gestiones externas también incluye las relaciones públicas que "Son el esfuerzo planeado para influenciar la opinión mediante el buen carácter y la ejecución responsable, basadas en una comunicación de dos direcciones mutuamente satisfactorias" (Center y Cutlip, 2001, p.5). Ostentando que las relaciones públicas tienen como propósito establecer líneas mutuas de comunicación, comprensión y aceptación entre una institución y su público.

Con respecto a la segunda variable y sus dimensiones acerca del Posicionamiento de la Actividad Económica se destacan los siguientes autores:

"El posicionamiento en términos generales se asocia con el lugar, situación o emplazamiento, ocupado por un objeto, individuo, idea, una institución, un servicio, ciudad, o cualquier otra realidad susceptible de ser colocada respecto a otras con las que se compara" (Carasila, 2001, p.3). Por ende al posicionamiento se la determina en la comunidad donde se desarrollan diferentes gestiones, destacando a la comunicación como un medio que permite el crecimiento de las actividades comerciales de los habitantes.

De allí se abarcan las leyes de posicionamiento como parámetros o guías para gestionar según las necesidades de una comunidad "La ley de liderazgo corresponde a las autoridades o representantes de una institución, así mismo la ley de enfoque consiste en plantear metas colectivas y la ley de exclusividad tiene como propósito una institución competitiva" (Ries y Trout, 2002, p.5). Es decir, la comunicación en las instituciones efectúa un rol significativo dentro de 
la comunidad, porque los habitantes logran organizarse en diferentes áreas permitiéndoles alcanzar sus metas u objetivos.

Definitivamente, la comunicación estará ligada a la economía ya que toda institución tiene planificaciones que cumplir, donde sus gestiones externas involucran a la comunicación para establecer vínculos con sus habitantes, los cuales se desarrollan en sus diferentes actividades comerciales, sociales y culturales, sin dejar de lado la finalidad principal que es posicionar económicamente a una comunidad mediante sus acciones.

\section{MATERIALES Y MÉTODOS}

El estudio sobre Gestión de Comunicación Externa para el posicionamiento de la actividad económica en la Parroquia Atahualpa el cual está basado en el enfoque mixto que asume una realidad subjetiva y dinámica que está compuesta de diferentes contextos, donde está sustentada bajo el análisis reflexivo de los significados. De acuerdo con Hernández et al. (2003) indica que:

Representa el más alto grado de integración o combinación entre los enfoques cualitativo y cuantitativo. Ambos se entremezclan o combinan en todo el proceso de investigación, o, al menos, en la mayoría de sus etapas (...) agrega complejidad al diseño de estudio; pero contempla todas las ventajas de cada uno de los enfoques (p.21).

Por ende, el propósito de la investigación es básica puesto que pretende determinar las Gestiones de Comunicación Externa del GAD Parroquial Atahualpa, este tipo de investigación también es conocida como "Fundamental, exacta o investigación pura, que se ocupa del objeto de estudio sin condicionar una aplicación inmediata, pero teniendo en cuenta que, a partir de sus resultados y descubrimientos, pueden surgir nuevos productos y avances científicos" (Cívicos y Hernández, 2007, p.159). Esto implica que el estudio ayudará a conocer las gestiones direccionadas al posicionamiento económico empleadas por la institución Parroquial.

Concerniente al diseño se estipulo a la investigación descriptiva que según los criterios de Bernal (2006), se "Muestran, narran o identifican hechos, situaciones, rasgos, características del objeto de estudio, en las que se guía de preguntas de 
investigación formuladas por el investigador donde se soporta en técnicas como la encuesta, entrevista, la observación y la revisión documental" (p.136). Es decir, mediante la investigación descriptiva se podrá especificar las variables dependiente e independiente que son: las Gestiones de Comunicación Externa y el Posicionamiento Económico en la Parroquia Atahualpa.

Por lo tanto, la fuente de investigación será documental donde el Comité Internacional de Editores de Revistas Biomédicas (2001), la definen como "El resultado de otras investigaciones, de reflexiones de teóricos, lo cual representa la base teórica del área objeto de investigación ya que el conocimiento se construye a partir de su lectura, análisis, reflexión e interpretación de dichos documentos" (p.2). De esta manera, la información recolectada a través de otros estudios permitirá el enriquecimiento de la investigación lo que definirá las gestiones externas utilizadas por el GAD Parroquial Atahualpa.

En relación con el método se estableció al inductivo que refiere a la acción de inducir mediante el razonamiento, consiste en obtener hechos que van desde lo particular a lo universal. Inicia desde la observación propia de las características de un fenómeno y adquiere conclusiones de carácter general. Así mismo el estudio acerca de la Gestión de Comunicación Externa que se llevará a cabo en el Gobierno Autónomo Descentralizado (GAD) Parroquial Atahualpa, ubicada en la Provincia de Santa Elena, institución que goza de autonomía política, administrativa y financiera que están regidos por los principios de solidaridad, integración y participación ciudadana. Con respecto a los criterios de inclusión y exclusión; en la inclusión se eligió a la Parroquia Atahualpa por ser una comunidad de poca extensión territorial en vía de desarrollo, en cuanto a la exclusión no se optó por otras parroquias ya que se ha visto el avance en áreas comunicacionales y económicas.

Por consiguiente, la población finita de la Parroquia Atahualpa es de aproximadamente 4,025 habitantes, donde la muestra está determinada por el cuerpo administrativo del GAD Parroquial Atahualpa conformada por veinte miembros en diferentes áreas de la institución, cabe recalcar que el GAD Parroquial no cuenta con un departamento de comunicación por tal razón el muestreo consta por los nueve funcionarios que la conforman los directivos 
electos por votación popular y los miembros pertenecientes a otros departamentos que manejan aspectos comunicacionales; siendo el muestreo no probabilístico por conveniencia el que "Permite seleccionar aquellos casos accesibles que acepten ser incluidos, esto fundamentado en la conveniente accesibilidad y proximidad de los sujetos para el investigador" (Otzen y Manterola, 2017, p.230). Esto significa que el número de sujetos a investigar es reducida ya que el investigador conoce las características y la accesibilidad del objeto de estudio, que mediante la recolección de información permitirá analizar e identificar la Gestión de Comunicación Externa y a su vez determinar su posicionamiento económico.

Es por ello que la técnica es la encuesta que puede "Ser utilizada como técnica o como método, la cual permite recolectar datos por medio de la interrogación que se realiza al encuestado con el propósito de que brinden la información requerida para la investigación” (López y Fachelli, 2015, p.18). Además, el instrumento a utilizar es el cuestionario que comúnmente es requerida en trabajos de investigación la cual "Consiste en un conjunto de preguntas presentadas y enumeradas en una tabla y una serie de posibles respuestas que el encuestado debe responder" (González, 2021, p.21). Cabe recalcar que las interrogantes del cuestionario están basadas en las varibles y dimensiones del tema de investigación, ajustándose a los objetivos de analizar y determinar la Gestión de Comunicación Externa en la Parroquia Atahualpa. El instrumento será validado por el Lcdo. Gerzón Alfredo Cochea Panchana y el Lcdo. Luis Alberto Iza Espinoza, profesionales expertos en comunicación. Este instrumento será aplicado en la primera semana de diciembre del presente año.

Por otra parte, las variables establecidas en el estudio en la que se detalla que la variable independiente corresponde a la Gestión de Comunicación Externa donde sus dimensiones son: La comunicación estratégica, la publicidad y las relaciones públicas, ya que sus categorías detallan: los medios de difusión de información, imagen institucional, recursos humanos y elaboración de vallas publicitarias. Además, la variable dependiente que trata del posicionamiento por lo cual sus dimensiones se desglosan del posicionamiento comunicacional y económico, así mismo se categorizan por la relación que tienen entre sí y sus recursos materiales. Por ultimo, los aspectos éticos que deben considerarse 
durante el proceso investigativo para no perjudicar o comprometer la imagen institucional, radican en la confidencialidad de información y el mantenimiento del anonimato e intimidad de los participantes, sin recurrir a la invación de la privacidad. También, la no coacción que reside en no obligar a las personas de una organización a ser partícipes de un estudio, prevaleciendo que es importante tener el consentimiento de los miembros para obtener información siendo transparentes en manejar correctamente los datos y, a su vez comunicativos para compartir los resultados con los colaboradores, así mismo el preservar los datos originales y esto implica que la información debe ser conservada por el investigador donde la finalidad es que el estudiante se involucre en esquemas investigativos.

\section{RESULTADOS}

En este apartado se presentan los datos hallados en la investigación, la misma que se abordó mediante un cuestionario de preguntas al cuerpo administrativo del GAD Parroquial Atahualpa, el cual está conformado por nueve miembros institucionales que a través de una serie de interrogantes se logró determinar las gestiones de comunicación externa para el posicionamiento de la actividad económica.

Tabla 1. Variable independiente Gestión de comunicación externa.

¿EI GAD Parroquial Atahualpa tiene vínculo con otras instituciones para realizar gestiones y planificaciones de sus diferentes acciones laborales?

\begin{tabular}{ccc}
\hline Escala & Frecuencia & Porcentaje \\
\hline Nunca & 0 & $0 \%$ \\
En ocasiones & 0 & $0 \%$ \\
Con frecuencia & 0 & $0 \%$ \\
Casi siempre & 2 & $22 \%$ \\
Siempre & 7 & $78 \%$ \\
\hline Total & $\mathbf{9}$ & $\mathbf{1 0 0} \%$
\end{tabular}

Fuente: Cuerpo administrativo del GAD Parroquial Atahualpa. Elaboración propia.

Figura 1. Variable independiente Gestión de comunicación externa. 


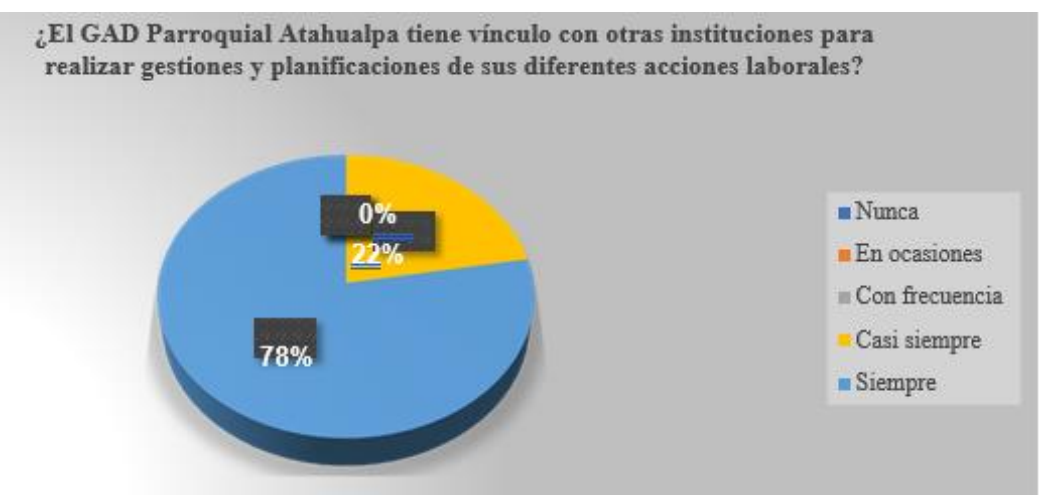

Fuente: Tabla de frecuencia. Elaboración propia.

Análisis: Los resultados del cuestionario se detallan de la siguiente manera: Casi siempre (2), que corresponde al 22\%; Siempre (7), que equivale al $78 \%$; En los ítems de: Nunca (0); En ocasiones (0) y Con frecuencia (0) que refleja al $0 \%$. En relación con el vínculo que ha mantenido el GAD Parroquial Atahualpa con otras instituciones se deduce que siempre manejan este tipo de lazos para realizar gestiones y planificaciones de sus diferentes acciones laborales.

Tabla 2. Variable independiente Gestión de comunicación externa.

¿EI GAD Parroquial Atahualpa utiliza estrategias comunicacionales como: ¿Difundir información a través de redes sociales Facebook, Instagram, WhatsApp y Twitter?

\begin{tabular}{ccc}
\hline Escala & Frecuencia & Porcentaje \\
\hline Nunca & 0 & $0 \%$ \\
En ocasiones & 0 & $0 \%$ \\
Con frecuencia & 3 & $33 \%$ \\
Casi siempre & 2 & $22 \%$ \\
Siempre & 4 & $45 \%$ \\
\hline Total & $\mathbf{9}$ & $\mathbf{1 0 0} \%$
\end{tabular}

Fuente: Cuerpo administrativo del GAD Parroquial Atahualpa. Elaboración propia.

Figura 2. Variable independiente Gestión de comunicación externa.

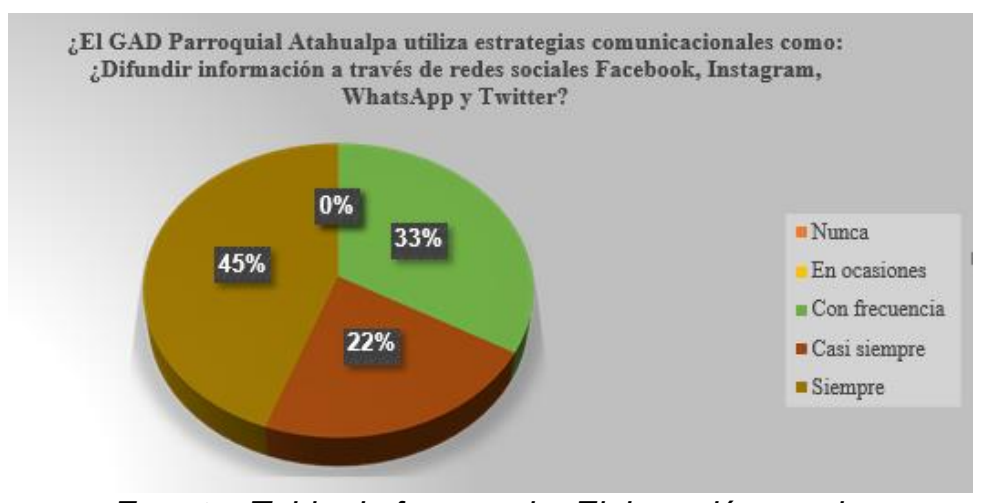

Fuente: Tabla de frecuencia. Elaboración propia. 
Análisis: Los resultados del cuestionario se detallan de la siguiente manera: Con frecuencia (3), con el 33\%; Casi siempre (2), equivalente al 22\%; Siempre (4), correspondiente al 45\%; En los ítems de: Nunca (0) y En ocasiones (0) que son el $0 \%$. Referente a las estrategias comunicacionales del GAD Parroquial Atahualpa se establece que siempre se utiliza las redes sociales de Facebook, Instagram, WhatsApp y Twitter como medio para difundir información.

Tabla 3. Variable independiente Gestión de comunicación externa.

¿EI GAD Parroquial Atahualpa elabora publicidades online para promocionar las actividades productivas de sus habitantes?

\begin{tabular}{ccc}
\hline Escala & Frecuencia & Porcentaje \\
\hline Nunca & 0 & $0 \%$ \\
En ocasiones & 0 & $0 \%$ \\
Con frecuencia & 0 & $0 \%$ \\
Casi siempre & 2 & $22 \%$ \\
Siempre & 7 & $78 \%$ \\
\hline Total & $\mathbf{9}$ & $\mathbf{1 0 0} \%$ \\
\hline
\end{tabular}

Fuente: Cuerpo administrativo del GAD Parroquial Atahualpa. Elaboración propia.

Figura 3. Variable independiente Gestión de comunicación externa.

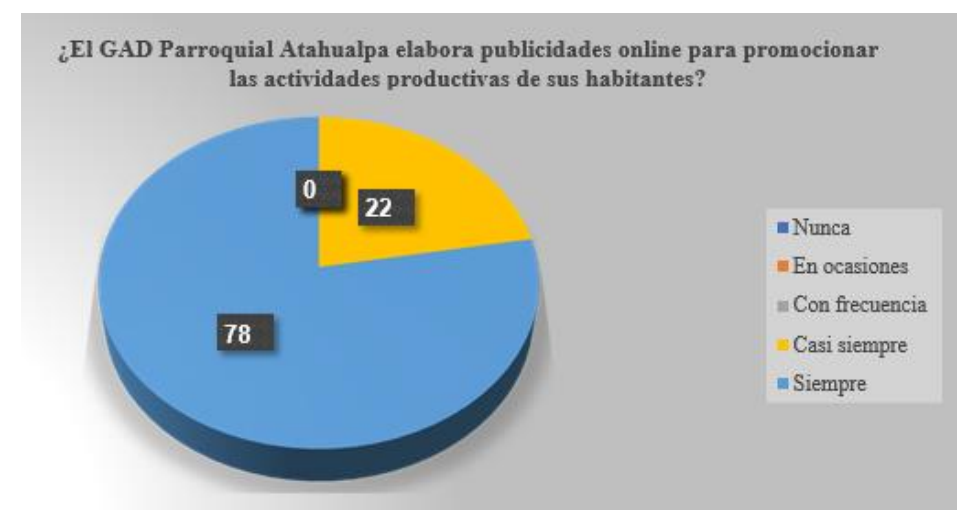

Fuente: Tabla de frecuencia. Elaboración propia.

Análisis: Los resultados del cuestionario se detallan de la siguiente manera: Casi siempre (2), que es el 22\%; Siempre (7), que corresponde al 78\%; En los ítems de: Nunca (0); En ocasiones (0) y Con frecuencia (0) equivalente al $0 \%$. Con respecto a las publicidades online que emplea el GAD Parroquial Atahualpa se determina que siempre se elaboran con el fin de promocionar las actividades productivas de sus habitantes. 
Tabla 4. Variable dependiente Posicionamiento de la actividad económica.

¿La Parroquia Atahualpa ha logrado posicionarse económicamente a través de sus gestiones externas en los últimos años?

\begin{tabular}{ccc}
\hline Escala & Frecuencia & Porcentaje \\
\hline Nunca & 0 & $0 \%$ \\
En ocasiones & 3 & $33 \%$ \\
Con frecuencia & 3 & $33 \%$ \\
Casi siempre & 3 & $34 \%$ \\
Siempre & 0 & $0 \%$ \\
Total & 9 & $100 \%$ \\
\hline
\end{tabular}

Fuente: Cuerpo administrativo del GAD Parroquial Atahualpa. Elaboración propia.

Figura 4. Variable dependiente Posicionamiento de la actividad económica.



Fuente: Tabla de frecuencia. Elaboración propia.

Análisis: Los resultados del cuestionario se detallan de la siguiente manera: Casi siempre (3), que corresponde al 34\%; Con frecuencia (3), que es el 33\%; En ocasiones (3), con el 33\%; En los ítems de: Nunca (0) y Siempre (0) que reflejan el $0 \%$. Con respecto al posicionamiento económico de la Parroquia Atahualpa en los últimos años se revela que casi siempre ha logrado posicionarse a través de sus gestiones externas.

Tabla 5. Variable dependiente Posicionamiento de la actividad económica.

¿EI GAD Parroquial Atahualpa gestiona eventos o ferias para que los habitantes puedan presentar sus actividades comerciales ganadería, artesanía y agricultura?

\begin{tabular}{ccc}
\hline Escala & Frecuencia & Porcentaje \\
\hline Nunca & 0 & $0 \%$ \\
En ocasiones & 0 & $0 \%$ \\
Con frecuencia & 0 & $0 \%$
\end{tabular}




\begin{tabular}{ccc} 
Casi siempre & 0 & $0 \%$ \\
Siempre & 9 & $100 \%$ \\
\hline Total & $\mathbf{9}$ & $\mathbf{1 0 0 \%}$ \\
\hline
\end{tabular}

Fuente: Cuerpo administrativo del GAD Parroquial Atahualpa. Elaboración propia.

Figura 5. Variable dependiente Posicionamiento de la actividad económica.

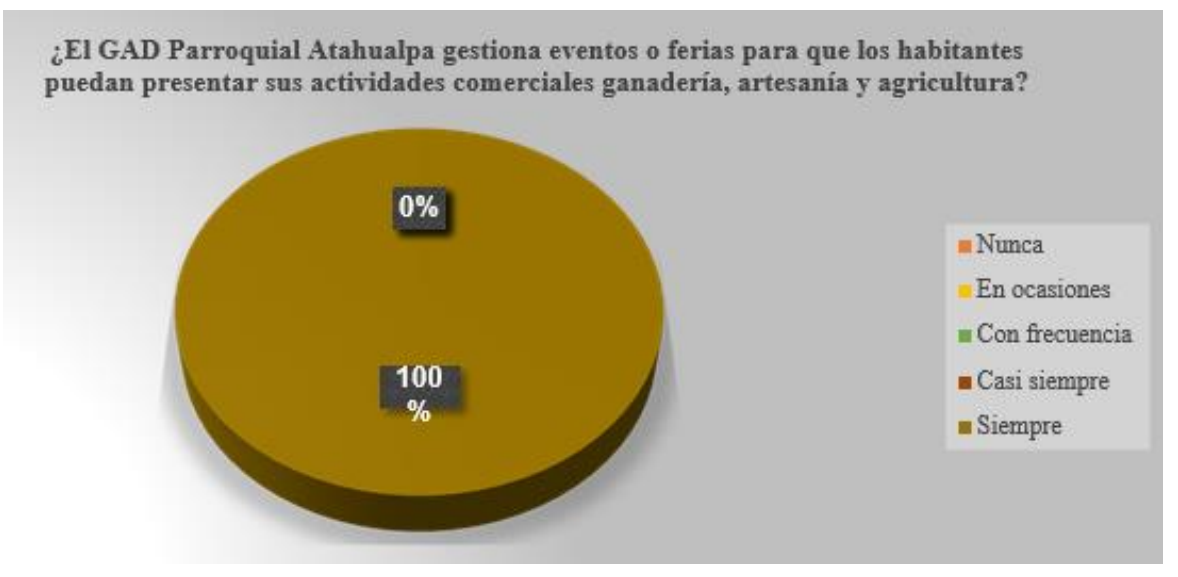

Fuente: Tabla de frecuencia. Elaboración propia.

Análisis: Los resultados del cuestionario se detallan de la siguiente manera: Siempre (9), equivalente al 100\%; En los ítems de: Nunca (0); En ocasiones (0); Con frecuencia (0) y Casi siempre (0) que corresponden al $0 \%$. En este aspecto el GAD Parroquial Atahualpa detalla que siempre ha gestionado eventos o ferias para que los habitantes puedan presentar sus actividades comerciales en la ganadería, artesanía y agricultura.

\section{DISCUSIÓN}

Posteriormente, los resultados hallados en este estudio responden a la pregunta de investigación científica que es la siguiente: ¿Cuáles son las gestiones de comunicación externa para el posicionamiento de la actividad económica en la Parroquia Atahualpa?, donde los resultados indican que el porcentaje mayor es del $78 \%$ que corresponde a la escala de Siempre, en cuanto al porcentaje menor que es del $0 \%$ perteneciente a la escala de Nunca; los datos alcanzados durante el proceso corroboran la respuesta a la pregunta de investigación. 
En relación con la evaluación de validez de los resultados adquiridos mediante la aplicación de técnicas e instrumentos, se logró establecer lo siguiente: El cuestionario instrumento empleado para la obtención de información permitió conocer de forma generalizada la problemática planteada en la investigación, de esta manera los resultados responden eficazmente al trabajo investigativo.

El análisis del cuestionario y de sus interrogantes dirigidas a conocer la Gestión de Comunicación Externa en la Parroquia Atahualpa siendo la problemática principal de estudio para determinar su posicionamiento económico, que mediante la recolección de información fue necesario el contacto directo entre el investigador y la institución; otros de los aspectos evidenciados es que existen necesidades comunicacionales en el personal administrativo del GAD Parroquial ya que tienen que capacitarse para gestionar estrategias de comunicación ante las exigencias y requerimientos de la comunidad. Con respecto a la triangulación en la cual se confirma la relación entre las variables de investigación, los objetivos y el marco teórico donde los resultados se describen en la discusión evidenciando el proceso investigativo.

La comparación de resultados de la pregunta uno, ¿El GAD Parroquial Atahualpa tiene vínculo con otras instituciones para realizar gestiones y planificaciones de sus diferentes acciones laborales?, en la que arrojo los siguientes datos: $78 \%$ Siempre y $0 \%$ Nunca, en la que se enlazan los criterios de Carlos (2020), donde trata de acciones particulares como la configuración de identidad corporativa, la gestación de espacios institucionales, en que deben estratificar sus comunicaciones para fortalecer sus relaciones y captar nuevos vínculos. Así mismo, Cáceres y Raymundo (2019), sugieren que la comunicación externa la empresa o institución ha de conocer el entorno general tanto político, social, cultural y económico, pero también debe conocer las tendencias de comportamiento que prevalecen en la sociedad donde quiere hacerse conocer.

Continuando con la comparación de resultados de la pregunta dos, ¿EI GAD Parroquial Atahualpa utiliza estrategias comunicacionales como: Difundir información a través de redes sociales Facebook, Instagram, WhatsApp y Twitter?, en la que se especifican los siguientes datos: $45 \%$ Siempre y $0 \%$ Nunca, donde se atañe el estudio de Ochoa (2019), que señala a las redes sociales como herramientas de gestión en la comunicación externa la cual 
genera interacción con el público, ya que las redes sociales permiten llegar con información directa y precisa.

Por consiguiente, la comparación de resultados de la pregunta tres, ¿El GAD Parroquial Atahualpa elabora publicidades online para promocionar las actividades productivas de sus habitantes?, en la que alcanzó los siguientes datos: $78 \%$ Siempre y 0\% Nunca, en la cual American Marketing Asociation define a la publicidad como la colocación de avisos y mensajes persuasivos, en tiempo o espacio que intentan informar y persuadir a los miembros de un mercado.

Así mismo, la comparación de resultados de la pregunta cuatro, ¿La Parroquia Atahualpa ha logrado posicionarse económicamente a través de sus gestiones externas en los últimos años?, en la que arrojo los siguientes datos: 34\% Casi siempre y $0 \%$ Nunca, donde se vincula a Gutiérrez (2015), ya que señala a la comunicación estratégica como herramienta de posicionamiento económico que muestra un panorama evolutivo de los medios de comunicación, dejando atrás el sentido unidireccional de la comunicación para abordar procesos de interacción y el intermediarismo.

Además, la comparación de resultados de la pregunta cinco, ¿EI GAD Parroquial Atahualpa gestiona eventos o ferias para que los habitantes puedan presentar sus actividades comerciales ganadería, artesanía y agricultura?, en la que se obtuvo los siguientes datos: $100 \%$ Siempre y 0\% Nunca, en este aspecto se considera a los autores Zavala y Lasso (2017), que indican la creación de estrategias de comunicación de los factores externos que permite el posicionamiento económico y los factores internos que tienen que ver con los costos de producción donde su diseño no está sujeta a exigencias de los demandantes.

Finalmente, la discusión presenta los resultados evidenciados durante el proceso investigativo y al mismo tiempo responde a la hipótesis de que la Gestión de Comunicación Externa utilizada por el GAD Parroquial Atahualpa permite alcanzar posicionamiento económico a través de sus actividades donde estas son consumadas en conjunto con sus habitantes; por consiguiente responde 
directamente a la pregunta problema la cual permitió mostrar información y datos verificados que ayuden a resolver problemas comunicacionales y que estas puedan solucionarse.

\section{CONCLUSIONES}

- La investigación aporta y fortalece a la comunidad científica, quienes son los encargados de ampliar, analizar y profundizar la información, siendo este estudio la base de futuras investigaciones acerca de la Gestión de Comunicación Externa direccionadas al posicionamiento económico de una comunidad, parroquia o sector.

- El trabajo investigativo estará al alcance de toda la comunidad de la Universidad Estatal Península de Santa Elena, donde se podrá acceder a esta información mediante el repositorio de la institución lo que proporcionará datos a otros estudios que se refieran a las Gestiones de Comunicación Externa para el Posicionamiento de la Actividad Económica.

- Este estudio se convertirá en fuente de investigación en la que tendrán acceso los profesores y estudiantes de la Carrera de Comunicación porque contribuye al conocimiento acerca de las Gestiones Externas que opera una institución en áreas comunicacionales el cual es importante para el desarrollo de una sociedad.

- El artículo científico responde a la pregunta problema acerca de: ¿Cuáles son las gestiones de comunicación externa para el posicionamiento de la actividad económica en la Parroquia Atahualpa? en la que se identificaron gestiones pertenecientes a la comunicación estratégica que corresponde al uso de redes sociales como medio para difundir información, la elaboración y creación de publicidades de las actividades comerciales de los habitantes en la Parroquia, también los vínculos que tiene con otras instituciones para el mejoramiento de proyectos y planificaciones laborales a pesar de no contar con un departamento de comunicación.

- La importancia de los resultados obtenidos de la investigación permite indicar que el GAD Parroquial Atahualpa necesariamente debe contar con su departamento de comunicación, donde el empleo de las gestiones externas no es totalmente efectuado por la falta de conocimiento y recursos. Con 
respeto al posicionamiento económico se evidencia que es una Parroquia en vía de desarrollo donde sus habitantes utilizan las redes sociales para compartir diferentes actividades comerciales.

\section{Recomendaciones}

- Se recomienda a la comunidad científica a realizar estudios en instituciones públicas de las parroquias de poca extensión territorial en vía de desarrollo porque se evidencian múltiples necesidades en el ámbito comunicacional y económico en el que se transforma en una problemática ante los cambios y el desarrollo de los habitantes.

- Se recomienda al GAD Parroquial Atahualpa y a sus directivos utilizar otras gestiones de comunicación externa el cual beneficia a los habitantes en sus diferentes actividades comerciales (ganadería, artesanía, agricultura) a posicionarse económicamente logrando fortalecer el vínculo entre las autoridades y la comunidad.

- Se recomienda al GAD Parroquial Atahualpa y a su cuerpo administrativo a realizar capacitaciones en el ámbito comunicacional referente al manejo de las gestiones de comunicación externa con respecto a la utilización de estrategias para que puedan estar preparados ante el avance y las exigencias que requiere una comunidad.

- Se recomienda a los profesores y estudiantes que conforman la comunidad universitaria UPSE a efectuar estudios y seguimientos a los avances de la comunicación en instituciones públicas sean estos en el contexto externo efectuando debates para el crecimiento científico.

\section{Agradecimiento}

Extiendo mi agradecimiento a Dios que siempre ha sido mi guía y mi mayor fortaleza en cada decisión y etapa de mi vida, así mismo agradezco a mi madre Clara Orrala por su amor y apoyo incondicional, a mi padre Wilmer Yagual por ser mi inspiración y el mejor compañero en cada aventura, a mis tías Katty Yagual y Mariuxi Figueroa por su confianza. Agradezco especialmente a mi abuela Bremilda Soriano por alentarme durante estos años de estudio. También, agradezco a mi novio Joao Yalamá por ser parte de este proceso y motivación 
en todos los aspectos. Finalmente, quiero agradecer a los docentes Lcdo. Luis Iza y Lcdo. Ángel Matamoros por sus aportes y sus conocimientos en la elaboración de este artículo.

\section{REFERENCIAS}

Barahona, A. (2019). Análisis de comunicación externa de la Dirección de Relaciones Internacionales de la Universidad Central del Ecuador.

Bernal. (2006). Marco Metodológico.

Biomédicas, C. I. (2001). Fundamentos de la investigación documental y la monografía .

Caceres, T., \& Raymundo, T. (2019). La relación entre la comunicación externa y la imagen institucional de la Municipalidad Distrital de Ticapampa Recuay.

Carasila, C. (2001). Importancia y Concepto del Posicionamiento una Breve Revisión Teórica. Red de Revistas Cientificas de América Latina, el Caribe, España y Portugal, 3.

Carlos, B. (2020). La gestión de la comunicación externa en A.J \& J.A Redolfi SRL: El Fortalecimiento del vínculo institucional con los clientes coorporativos.

Center, \& Cutlip. (2001). Las Relaciones Públicas: Herramienta fundamental en la creación y mantenimiento de la identidad e imagen corporativa. Primera Revista Electrónica en América Latina Especializada en Comunicación, 5.

Cívicos, \& Hernández. (2007). La investigación aplicada: una forma de conocer las realidades con evidencia científica.

Cortez, P. (2011). Las relaciones públicas y su incidencia en el funcionamiento de la comunicación interna y externa de AGUAPEN S.A periodo 20102011.

González, J. L. (2021). Técnicas e instrumentos de investigación científica.

Gutiérrez, G. (2015). La comunicación estratégica como herramienta de posicionamiento en negocios familares. Estudio de caso Gilisburger.

Hernández, Fernández, \& Baptista. (2003). Los diseños de método mixto en la investigación en educación: Una experiencia concreta. 21.

López, \& Fachelli, R. y. (2015). Técnicas e instrumentos de investigación científica. 
Ochoa, Y. (2019). Las redes sociales de la Universidad Nacional de Chimborazo como herramienta de gestión en la comunicación externa, período octubre 2017 marzo 2018.

Otzen, T., \& Manterola, C. (2017). Técnicas de muestro sobre una población a estudio.

Ries, \& Trout. (2002). El concepto del posicionamiento desde la perspectiva de profesionales del marketing y el consumidor Caleño, una investigación exploratoria.

Sicoli, A. (2016). Gestión de la comunicación externa de la empresa de transporte A\&A de la provincia de Córdoba.

Suárez, E. L. (2012). Las relaciones públicas y su influencia en la sociabilidad comunicativa entre el Gobierno Autónomo Descentralizado Parroquial de Chanduy y sus Comunidades durante el período 2011 - 2012.

Thompson, I. (2005). Definición de Publicidad.

Tironi, E., \& Cavallo, A. (2007). Comunicación Estratégica vivir en un mundo de señales.

Zavala, A. H., \& Lasso, M. d. (2017). Pymes como modelo económico en la creación de estrategias de comunicación. 